\title{
ACTITUD EMPRENDEDORA DE ESTUDIANTES DEL ÚLTIMO AÑO ACADÉMICO EN UNA UNIVERSIDAD EN ECUADOR
}

\section{ENTERPRISING ATTITUDE OF STUDENTS OF THE LAST YEAR OF ACADE- MIC IN A UNIVERSITY IN ECUADOR}

\author{
Carlos A. Murillo-Barrera', Miguel A. Bustamante U. ${ }^{2}$, Cristian L. Vidal-Silva ${ }^{3}$, Roberto M. Campos ${ }^{\star 2}$. \\ 1 Maestría en Administración de Empresas, Universidad Católica Santiago de Guayaquil, Guayaquil, Ecuador. E-mail: carlosandresmurillobarrera@gmail.com \\ 2 Facultad de Economía y Negocios, Universidad de Talca, Avenida Lircay S/N, Talca-Chile. E-mail: mabu@utalca.cl; rcampos@utalca.cl \\ 3 Ingeniería Civil Informática, Escuela de Ingeniería, Campus Rodelillo, Universidad Viña del Mar, Agua Santa 7055, Viña del Mar-Chile. E-mail: cristian.vidal@uvm.cl
}

RESUMEN

El presente artículo analiza la actitud de emprendimiento de estudiantes universitarios de una institución de educación superior en Ecuador. Se realizó un estudio descriptivo transversal a una muestra de estudiantes universitarios regulares de una Universidad del Estado que cursan el último año de la carrera de ingeniería en negocios. Se aplicó un cuestionario estructurado con preguntas en escala tipo Likert de 5 puntos. Se realizó análisis de clúster jerárquico, además se realizó un análisis de regresión para determinar incidencias entre las variables. No se aprecia una mayor predisposición emprendedora entre los estudiantes y no se muestran convencidos de perseverar en dicha competencia. Además, quienes cuentan con familiares emprendedores, son más adversos al riesgo. Se verifica también un aceptable nivel de control interno y conciencia de que las acciones generan resultados consecuentes. En el grupo entrevistado más joven existe una ligera predisposición a la idea del negocio propio. Aunque existe temor a la inversión de sus ahorros, sin embargo, se aprecia que todos los grupos estarían dispuestos a dar todo de si por un negocio propio, sin embargo, la variable más significativa e inductora del emprendimiento es la familia.

Palabras clave: actitud emprendedora; universidad; emprendimiento; vida académica.
ABSTRACT

The present article analyzes the attitude of university students' of an institution of superior education emprendimiento in Ecuador. She/he was carried out a traverse descriptive study to a sample regular university students of a University of the State that you/they study the last year of the engineering career in business. A questionnaire was applied structured with questions in scale type Likert of 5 points. She/he was carried out analysis of hierarchical clúster, she/he was also carried out a regression analysis to determine incidences among the variables. A bigger venturesome bias is not appreciated among the students and they are not shown convinced of persevering in this competition. Also who count with family venturesome, they are more adverse to the risk. It is also verified an acceptable level of internal control and it makes aware that the actions generate consequent results. In the youngest interviewed group a slight bias exists to the idea of the own business. Although fear exists to the investment of its savings, however, it is appreciated that all the groups would be willing to give everything of if for an own business, however, the most significant variable and inductora of the emprendimiento are the family.

Keywords: enterprising attitude; university; entrepreneurship; academic life. 


\section{INTRODUCCIÓN}

Las personas que deciden convertirse en empresarios suele tener entre 25 y 45 años, presentan agilidad física y mental, son hábiles logrando nuevos aprendizajes y poseen menos responsabilidades (Cheril, 2018), lo que les permite emprender, además provienen de distintos niveles socioeconómicos y culturales, así como también presentan diferencias de género (Chinyamurindi, 2016).

En términos de género, se observa que las mujeres incrementan su presencia (Dastourian, et al 2017) evidenciando rasgos característicos tales como agresividad, determinación, comunicación, dirección, independencia, ambición, responsabilidad, innovación, creatividad y propensión para tomar riesgos, además de proactividad y prontitud para el cambio (Ferraz et al., 2014).

En general, el ambiente universitario influye sobre los estudiantes (Arquero y Tejero, 2006) en particular sobre la intención emprendedora desarrollando capacidades como la de asumir riesgo, la predisposición a la acción y la valoración de la propia auto eficacia (Salati et al., 2018), sin embargo, de acuerdo con el trabajo de Soria et al. (2016), un curso universitario de emprendimiento puede afectar negativamente la intención emprendedora (IE) de los estudiantes. En principio, porque el emprendimiento puede ser comprendido como parte de una herencia familiar (Klein, 2016) así como derivar de la red familiar y social de padres y parientes que motivan el espíritu emprendedor y son fuentes principales de relación con el mundo de los negocios (Contreras et al., 2017)

La intención emprendedora de los estudiantes se observa en la capacidad de asumir riesgos (Dastourian, et al., 2017), sin embargo, las normas subjetivas de presión social juegan un rol inverso aunque de magnitud pequeña sobre la conducta emprendedora (Naia et al., 2017). Por otra parte, la experiencia y formación previa de un emprendedor resulta ser un factor importante en la conducta (Caro-Gon- zález et al., 2016), que en el caso de las universidades colombianas, es más bien teórica y sin la suficiente consideración a la práctica, aunque con esporádicos contactos con empresarios a través de conferencias (Soria-Barreto, et al., 2017).

En lo fundamental, el emprendimiento presenta algunas características clave (Chinyamurindi, 2016), entre allas la motivación y la capacidad para enfrentar el riesgo, la tolerancia a la ambigüedad y el espíritu de negocios, como talentos endógenos que facultan a la persona hacia el emprendimiento (Soria, 2016): Complementariamente, es preciso tener presente que el trabajo del empresario implica indagar, investigar y descubrir las múltiples combinaciones y recombinaciones de los recursos de qué dispone y de los que requiere, para incrementar sus retornos o anular las pérdidas (Klein, 2016). Todo esto propicia, en definitiva, el esfuerzo individual hacia el logro y que conceptualmente se denomina, conducta emprendedora (Araya y Pedreros, 2013).

Profundizando en esta línea de análisis, en general, quienes emprenden tienen habilidades, como las de identificar oportunidades y, a través ellas, servir alguna necesidad social que les reporte algún beneficio (Castillo y Alejandro, 2016). Sin embargo, esto no es directo ni inmediato, sino que se alcanza mediante el ejercicio del pensamiento innovador y de su capacidad para auto motivarse, activar procesos exógenos y gestionar los recursos para agregar valor a la respuesta que ofertan a la sociedad (Manyaka - Boshielo, 2017). Para ello el emprendedor, debe aprender a lidiar con el riesgo, la incertidumbre y la complejidad (Angulo, 2016), lo cual requiere y eventualmente exige, a nivel de las Universidades, la necesidad de desarrollar las competencias de sus estudiantes para habilitarlos para emprender, primero en el aula y luego, produciendo un ecosistema universitario protegido destinado al aprendizaje ( $\mathrm{Ar}$ quero y Tejero, 2006).

Sin embargo, por lo general los miembros de las facultades son líderes en el aula pero no contribuyen necesariamen- 
te de la misma manera a desarrollar el espíritu emprendedor que se precisa, en consecuencia, parece necesario transmitir al estudiante de manera comprensiva, la volatilidad del mundo de los negocios (Castillo y Alejandro, 2016), transmitiéndoles la experiencia de terceros por medio de las historias de vida de algunos empresarios, que muestran, promueven conductas exitosas y enseñan el camino (Chinyamurindi, 2016)

Por otra parte, las personas por su propia naturaleza humana, buscan instintivamente situarse en espacios controlados o seguros (Araya y Pedreros, 2013) y por lo mismo, se enfrenta a la necesidad de tener bajo control la fuente de su destino la que es conocida como locus de control o control interno (Bibiano et al., 2016). Gatillando un proceso donde cada individuo, aunque estimulado por sus singulares necesidades o expectativas, trata de satisfacer sus necesidades de logro para lo cual es preciso, que desarrollen la capacidad endógena de tomar el mando, así como también fortalecer sus rangos de tolerancia a la ambiguedad (Muñoz-Osuna, 2016). De esta forma se comienza a diseñar en cada individuo la competencia compleja del emprendimiento que, unida al apoyo familiar y aceptación de la comunidad, resultan significativos en la generación de un espíritu emprendedor sostenible (Bignotti y Le Roux, 2016)

La tolerancia a la ambigüedad habilita al individuo a afrontar situaciones confusas y de alta incertidumbre, sin tener que optar por decisiones precipitadas (Arquero y Tejero, 2006), lo cual significa que el emprendedor es capaz de asimilar el contexto de manera eficaz (Rodriguez, 2015). Es aquí dónde la universidad tiene bastante que aportar, por una parte, reproduciendo un ambiente en el que los estudiantes participen de juegos de simulación comercial y talleres de relación empresarial y, por otra, generando un armazón protegido que les vincule con el ambiente de negocios, pero desde la perspectiva segura del espacio académico que le proporciona la Universidad (Farrington et al., 2012).
Las cualidades emprendedoras tales como innovación, positivismo, adaptabilidad y liderazgo (Pedraja Rodríguez, 2018), y muchas otras, han sido incluida entre las competencias factibles de ser desarrolladas por la academia, potenciando la propensión emprendedora del aprendiz a partir del tipo de escuela, ambiente y formación pertinente al que se exponga para desarrollar sus cualidades de emprendimiento (Mahadea et al., 2011)

Sobre la base de lo analizado en este artículo, se busca interpretar las diferentes actitudes emprendedoras que se presentan en los estudiantes que cursan el último año de la carrera de ingeniería en negocios en una Universidad Estatal de Ecuador, mediante el análisis de las características, necesidad de logro, propensión al riesgo, control interno percibido, tolerancia a la ambigüedad y espíritu emprendedor.

\section{METODOLOGÍA}

El estudio fue no experimental adoptando el enfoque cuantitativo, correlacional y de asociación de variables, debidamente operacionalizadas, para analizar la incidencia de las independientes y determinar el carácter explicativo sobre la variable dependiente (Hernández, et al. , 2006). La población seleccionada fue de alumnos regulares de una Universidad del Estado, del último año de la carrera de ingeniería en negocios, secciones diurna y nocturna que totaliza 62 estudiantes. El ámbito geográfico del estudio, se circunscribe a la provincia del Guayas, Ecuador.

El periodo de recogida de datos fue el mes de julio del año 2017, mediante la aplicación de un cuestionario estructurado (Hernández, et al., 2006) con preguntas escogidas, depuradas y adaptadas al entorno académico ecuatoriano, medidas en escala tipo Likert (Likert, 1932) de 5 puntos unidireccional, dónde 1 indica que está totalmente en desacuerdo y 5 que está totalmente de acuerdo. El análisis de datos se realizó previa generación de una base de datos en SPSS, (Statical 
Package for Social Science) en su versión 22.

El trabajo de campo fue realizado por entrevistadores debidamente entrenados con una duración promedio de respuesta fue de 30 a 40 minutos. El levantamiento de los datos se realizó entre los meses de marzo y mayo de 2017. Los cuestionarios fueron aplicados de acuerdo con los criterios de anonimato y confidencialidad.

\section{DEFINICIÓN Y UNIDADES DE MEDIDA DE LAS VARIABLES}

La tabla 1 detalla las variables y escala de medición de las variables.

Tabla 1: Definición de variables y unidades de medida

\begin{tabular}{llll}
\hline Edad & Medida en años. & Género & Escala dicotómica. \\
\hline Formación & Semestre que se cursa. & $\begin{array}{l}\text { Experiencia } \\
\text { Laboral }\end{array}$ & $\begin{array}{l}\text { Número de años que haya trabajado } \\
\text { el estudiante. }\end{array}$ \\
\hline $\begin{array}{l}\text { Antecedentes } \\
\text { familiares }\end{array}$ & $\begin{array}{l}\text { Número de familiares directos del } \\
\text { estudiante que fueran empresarios. }\end{array}$ & $\begin{array}{l}\text { Necesidad de } \\
\text { logro }\end{array}$ & Escala 1-5. \\
\hline $\begin{array}{l}\text { Tolerancia a la } \\
\text { ambigüedad }\end{array}$ & Escala 1 a 5. & $\begin{array}{l}\text { Propensión al } \\
\text { riesgo }\end{array}$ & Escala 1-5. \\
\hline Control interno & Escala 1 a 5. & $\begin{array}{l}\text { Espíritu } \\
\text { emprendedor }\end{array}$ & Escala 1-5. \\
\hline
\end{tabular}

Para el análisis de las variables se aplicó el método de clúster jerárquico (Pérez, 2009), para agrupar y formar conglomerados homogéneos y definir clasificaciones pertinentes. Se tuvo sin embargo en cuenta que el análisis de clúster es descriptivo, ateórico y no inferencia y tiene el carácter de exploratorio, en consecuencia no ofrece soluciones únicas y, las soluciones que se obtienen dependen de las variables consideradas y del método de análisis de clúster empleado (Hernández, et al., 2006).

\section{ANÁLISIS DE REGRESIÓN}

De acuerdo con la metodología de análisis de regresión lineal múltiple (Gujarati y Portes, 2009), se generó una variable continua denominada emprendimiento mediante la sumatoria de las dimensiones necesidad, tolerancia, riesgo, control y experiencia. Se utilizó el método de mínimos cuadrados ordinarios MCO con el objetivo de asumir la eventual interdependencia de la conducta emprendedora respecto de las variables demográficas de control que pudiesen resultar correlacionadas (Lloret, et al., 2014).
Las variables independientes para el análisis fueron las demográficas, sexo, edad, semestre, experiencia laboral y familia. De éstas cinco variables, las correspondientes a sexo y semestre fueron categorizadas como dicotómicas, femenino y masculino; semestre1 y semestrez respectivamente.

\section{RESULTADOS}

De acuerdo con el análisis de clúster jerárquico (Tabla 2 ) se dividió la población en tres grupos para obtener un análisis consistente.

De las variables sociodemográficas no se aprecia una mayor diferencia por estratos. La edad bordea los 23 años, el género es mayormente femenino $(\geq 1)$, en general están cursando el $7^{\circ}$ semestre y declaran una experiencia media de 2,5 años. Los familiares empresarios resultan ser un ítem relevante puesto que se ubica entre o,25 y o,77 puntos, confirmando la existencia de a lo menos un familiar, especialmente en el estrato 1. 
Tabla 2: Descriptores de los estratos

\begin{tabular}{ccccccc}
\hline $\begin{array}{c}\text { Articulación Media } \\
\text { (entre grupos) }\end{array}$ & $\begin{array}{c}\text { Edad del } \\
\text { estudiante }\end{array}$ & $\begin{array}{c}\text { Género del } \\
\text { estudiante }\end{array}$ & $\begin{array}{c}\text { Semestre en que } \\
\text { se encuentra el } \\
\text { estudiante }\end{array}$ & $\begin{array}{c}\text { Años de experiencia } \\
\text { laboral del estudiante }\end{array}$ & $\begin{array}{c}\text { Familiares } \\
\text { emprendedores que } \\
\text { tiene el estudiante }\end{array}$ \\
\hline \multirow{3}{*}{1} & $\mathrm{~N}$ & 22 & 22 & 22 & 22 & 22 \\
& Media & 23,73 & 1,27 & 7,50 & 2,27 & 77 \\
& $\%$ & 35,5 & 35,5 & 35,5 & 35,5 & 35,5 \\
\cline { 2 - 7 } 2 & $\mathrm{~N}$ & 20 & 20 & 20 & 20 & 25 \\
& Media & 23,85 & 1,55 & 7,40 & 2,80 & 32,3 \\
\cline { 2 - 7 } 3 & $\%$ & 32,3 & 32,3 & 32,3 & 32,3 & 35 \\
& $\mathrm{~N}$ & 20 & 20 & 20 & 20 & 32,3 \\
\multirow{3}{*}{ Total } & Media & 23,95 & 1,40 & 7,45 & 32,3 & 62 \\
& $\%$ & 32,3 & 32,3 & 32,3 & 62 &, 47 \\
& $\mathrm{~N}$ & 62 & 62 & 62 & 2,63 & 100,0 \\
\hline
\end{tabular}

Complementariamente, desde las percepciones recogidas de los estudiantes (tabla 3) se observa que existe un nivel alto de necesidad de logro y es posible afirmar que los estudiantes tienen el deseo de mejorar y alcanzar las metas que se han propuesto.

A lo menos los estudiantes manifiestan la intención de asumir el emprendimiento, sin embargo, al analizar las últimas dos preguntas, se observa un ligero decremento con relación a las anteriores, por cuanto, aun cuando dicho decremento no es significativo, se manifiesta temor e incertidumbre al momento de tomar decisiones de emprendimiento.

Tabla 3: Características personales de emprendimiento

\begin{tabular}{|c|c|c|c|c|c|c|}
\hline Cluster & Medidas & $\begin{array}{c}\text { Me gusta } \\
\text { destacar en mi } \\
\text { trabajo o estudios }\end{array}$ & $\begin{array}{l}\text { Soy muy } \\
\text { minucioso en las } \\
\text { tareas que } \\
\text { realizo }\end{array}$ & $\begin{array}{c}\text { Me gusta trabajar } \\
\text { para estar } \\
\text { siempre entre los } \\
\text { mejores } \\
\text { diariamente }\end{array}$ & $\begin{array}{l}\text { Soporto bien las } \\
\text { situaciones } \\
\text { confusas y poco } \\
\text { claras o de } \\
\text { indefinición }\end{array}$ & $\begin{array}{l}\text { Manejo a la } \\
\text { perfección las } \\
\text { situaciones de } \\
\text { incertidumbre }\end{array}$ \\
\hline \multirow{4}{*}{1} & $\mathrm{~N}$ & 22 & 22 & 22 & 22 & 22 \\
\hline & Media & 4,77 & 4,55 & 4,64 & 4,32 & 4,23 \\
\hline & $\%$ & 35,5 & 35,5 & 35,5 & 35,5 & 35,5 \\
\hline & $\mathrm{N}$ & 20 & 20 & 20 & 20 & 20 \\
\hline \multirow[t]{3}{*}{2} & Media & 4.45 & 4,45 & 4,45 & 4,00 & 4,30 \\
\hline & $\%$ & 32,3 & 32,3 & 32,3 & 32,3 & 32,3 \\
\hline & $\mathrm{N}$ & 20 & 20 & 20 & 20 & 20 \\
\hline \multirow[t]{2}{*}{3} & Media & 4,65 & 4,60 & 4,70 & 4,55 & 4,40 \\
\hline & $\%$ & 32,3 & 32,3 & 32,3 & 32,3 & 32,3 \\
\hline \multirow[t]{3}{*}{ Total } & $\mathrm{N}$ & & & 62 & 62 & \\
\hline & Media & 4,63 & 4,53 & 4,60 & 4,29 & 4,31 \\
\hline & $\%$ & 100,0 & 100,0 & 100,0 & 100,0 & 100,0 \\
\hline
\end{tabular}

Las preguntas relacionadas con las variables de propensión al riesgo y control interno están reflejadas en la tabla 4. Según los resultados de las dos primeras preguntas que corresponden a riesgo, se determina que, además de existir un comportamiento de los estudiantes no totalmente convencidos con el emprendimiento, también se aprecia que los miembros del primer grupo, de aparentemente menor edad y con una ligera minoría en la experiencia laboral pero con un índice mayor de familiares emprendedores, son más adversos al riesgo.

Se puede afirmar así mismo, que probablemente, por las experiencias percibidas de sus familiares directos, los estudiantes optan progresivamente por una posición casi neutral frente al riesgo en el que no están ni a favor ni en contra del mismo. Así también se aprecia que existe un acep- 
table nivel de control interno, es decir, que existe conciencia de que, de acuerdo con las decisiones tomadas, existirá un resultado derivado de sus acciones del cual deberán hacerse cargo.

De acuerdo con los resultados de la tabla 4 , se puede apreciar además, que nuevamente en el grupo más joven también existe una ligera diferencia con los demás en las preguntas referidas a tener empresa propia y a la preferencia de ser empleado o emprendedor, es decir, a la idea del propio negocio, y sobre todo en el temor a la inversión de sus ahorros.

Con respecto a la tercera pregunta en relación con el esfuerzo y tiempo a dedicar, se aprecia que no existe mayor diferencia y que todos estarían dispuestos a dar todo de si por un negocio propio.

Tabla 4: Caracterización de los estudiantes

\begin{tabular}{|c|c|c|c|c|c|}
\hline \multicolumn{6}{|c|}{ Riesgo } \\
\hline Clúster & Medidas & $\begin{array}{l}\text { Me parece que si } \\
\text { no asumo } \\
\text { riesgos me } \\
\text { quedaré } \\
\text { estancado/a }\end{array}$ & $\begin{array}{l}\text { Las personas que asumen } \\
\text { riesgos tienen más } \\
\text { probabilidades de salir } \\
\text { adelante que las que no } \\
\text { se arriesgan }\end{array}$ & $\begin{array}{c}\text { Me gusta planear a } \\
\text { tiempo las actividades } \\
\text { que realizo }\end{array}$ & $\begin{array}{l}\text { Nunca pospongo asuntos } \\
\text { importantes para "un } \\
\text { momento mejor" aunque } \\
\text { esté presionado por el } \\
\text { tiempo }\end{array}$ \\
\hline \multirow{4}{*}{1} & $\mathrm{~N}$ & 22 & 22 & 22 & 22 \\
\hline & Media & 3,86 & 3,55 & 4,59 & 4,18 \\
\hline & $\%$ & 35,5 & 35,5 & 35,5 & 35,5 \\
\hline & $\mathrm{N}$ & 20 & 20 & 20 & 20 \\
\hline \multirow[t]{2}{*}{2} & Media & 4,20 & 4,20 & 4.70 & 4,10 \\
\hline & $\%$ & 32,3 & 32,3 & 32,3 & 32,3 \\
\hline \multirow{3}{*}{3} & $\mathrm{~N}$ & 20 & 20 & 20 & 20 \\
\hline & Media & 4,40 & 4.50 & 4.55 & 4,40 \\
\hline & $\%$ & 32,3 & 32,3 & 32,3 & 32,3 \\
\hline \multirow{4}{*}{ Total } & $\mathrm{N}$ & 62 & 62 & 62 & 62 \\
\hline & Media & 4,15 & 4,06 & 4,61 & 4,23 \\
\hline & $\%$ & 100,0 & 100,0 & 100,0 & 100,0 \\
\hline & \multicolumn{5}{|c|}{ Espíritu emprendedor } \\
\hline \multirow[t]{6}{*}{ Cluster } & Medidas & Pienso en la & La idea de tener mi propio & Si decido crear mi & \\
\hline & & posibilidad de & negocio frente a ser un & propio negocio estaría & \\
\hline & & $\begin{array}{l}\text { algún día tener } \\
\text { mi propio }\end{array}$ & $\begin{array}{l}\text { asalariado de una } \\
\text { empresa me parece }\end{array}$ & $\begin{array}{l}\text { dispuesto a dedicar } \\
\text { todo el tiempo }\end{array}$ & Para noder tener mi propin \\
\hline & & negocio & mucho más interesante & necesario y realizar & negocio estaría dispuesto \\
\hline & & & & todo mi esfuerzo & a invertir mis ahorros \\
\hline & $\mathrm{N}$ & 22 & 22 & 22 & 22 \\
\hline \multirow[t]{3}{*}{1} & Media & 4,41 & 4,05 & 4,45 & 3,64 \\
\hline & $\%$ & 35,5 & 35,5 & 35,5 & 35,5 \\
\hline & $\mathrm{N}$ & 20 & 20 & 20 & 20 \\
\hline \multirow[t]{2}{*}{2} & Media & 4,75 & 4,45 & 4,40 & 4,15 \\
\hline & $\%$ & 32,3 & 32,3 & 32,3 & 32,3 \\
\hline \multirow{3}{*}{3} & $\mathrm{~N}$ & 20 & 20 & 20 & 20 \\
\hline & Media & 4,65 & 4,55 & 4,65 & 4,20 \\
\hline & $\%$ & 32,3 & 32,3 & 32,3 & 32,3 \\
\hline \multirow{3}{*}{ Total } & $\mathrm{N}$ & 62 & 62 & 62 & 62 \\
\hline & Media & 4,60 & 4,34 & 4,50 & 3,98 \\
\hline & $\%$ & 100,0 & 100,0 & 100,0 & 100,0 \\
\hline
\end{tabular}

\section{ANÁLISIS DE REGRESIÓN}

Al analizar los resultados de la regresión lineal múltiple (Tala 5), se observa que las variables dicotómicas Masculino y Semestrez no reportan índices suficientes para determinar la relación, lo que se puede deber al comportamiento errático de sus frecuencias.

De acuerdo con lo indicado, el compor- tamiento descrito de las variables se ve reflejado en los valores entregados por el índice $\mathrm{R}(\mathrm{R} 2=0,101)$, el cual resulta considerablemente bajo seguido del hecho que la prueba $F(F$ critico $=1,258)$ reporta un valor menor al valor $\mathrm{F}$ más próximo disponible en tabla $\left(\mathrm{F}=2,40 \mathrm{df} 1=5, \mathrm{df}_{2}=50\right.$; motivo por el cual los resultados del análisis de estas variables no resultan significativos.

La no significación determinada, puede 
ser causada en parte, porque no reflejan con precisión la relevancia de sus relaciones dentro del análisis; por otra parte, por el hecho de que algunas de las variables analizadas sólo cuentan con dos reactivos para ser medidas y, tercero, porque eventualmente, los 62 contactos recogidos cumplen levemente con el criterio de muestra mínima de proporción (n/p, 5:1; Lloret et al., 2014).
Por otra parte, las pruebas $t$, para las variables Edad, Femenino, Masculino, y Experiencia no son significativas en el análisis por lo que tampoco estas describen el modelo de forma independiente. Sin embargo, se observa que la variable Familiares alcanza un nivel de significancia puesto que entrega un índice p-valor cercano al $10 \%$.

Tabla 5: Estimaciones de los coeficientes Regresión Lineal Múltiple

\begin{tabular}{|c|c|c|c|c|}
\hline & Estimador & Error Estándar & t value & $\operatorname{Pr}(>|t|)$ \\
\hline (Intercepto) & 54.00861 & 10.19497 & 5.298 & $2.04 \mathrm{e}-06^{* * *}$ \\
\hline Edad & 0.07853 & 0.45665 & 0.172 & 0.8641 \\
\hline Femenino & -1.12889 & 1.12757 & -1.001 & 0.3211 \\
\hline Masculino & NA & NA & NA & NA \\
\hline Semestre1 & 0.91552 & 1.10207 & 0.831 & 0.4097 \\
\hline Semestre2 & NA & NA & NA & NA \\
\hline Experiencia & 0.20619 & 0.41162 & 0.501 & 0.6184 \\
\hline Familiares & 1.22170 & 0.66473 & 1.838 & $0.0714^{*}$ \\
\hline Error residual estandarizado & \multicolumn{4}{|l|}{$4,158(56 \mathrm{df})$} \\
\hline R-cuadrado & \multicolumn{4}{|l|}{0,101} \\
\hline R-cuadrado Ajustado & \multicolumn{4}{|c|}{0,02073} \\
\hline F estadístico & \multicolumn{4}{|c|}{$1,258$ (5 df1, $56 \mathrm{df} 2)$ p-value 0,2948} \\
\hline & Media & \multicolumn{2}{|c|}{ Desviación típica } & $\mathrm{N}$ \\
\hline Emprendimiento & 56,82 & \multicolumn{2}{|c|}{4,202} & 62 \\
\hline Edad & 23,84 & \multicolumn{2}{|c|}{1,691} & 62 \\
\hline Femenino & 0,60 & \multicolumn{2}{|c|}{0,495} & 62 \\
\hline Masculino & 0,40 & \multicolumn{2}{|c|}{0,495} & 62 \\
\hline Semestre1 & 0,55 & \multicolumn{2}{|c|}{0,502} & 62 \\
\hline Semestre2 & 0,45 & \multicolumn{2}{|c|}{0,502} & 62 \\
\hline Experiencia & 2,63 & \multicolumn{2}{|c|}{1,649} & 62 \\
\hline Familiares & 0,47 & \multicolumn{2}{|c|}{0,804} & 62 \\
\hline
\end{tabular}

Sin perjuicio de lo indicado, la muestra de 62 contactos permite superar el mínimo de 50 casos (Lloret et al., 2014) lo que hace factible determinar algunos resultados del análisis de regresión múltiple, lográndose calcular los coeficientes que definen la ecuación lineal del constructo de emprendimiento (1), en la que se muestras los parámetros estimados de las variables relacionadas.

Emprendimiento $=54,0068+0,0785$ Edad $-1,1289$ Femenino +o,9155Semestre1+o,2062Experiencia+1,2217Familiares

La fórmula (1) muestra que la variable Familiares, es la única que demostró tener algún grado de significancia dentro del modelo en tanto y en cuanto alcanza un índice beta alto y significativo $(1,2217)$. Los resultados del presente estudio permiten, en consecuencia, arribar a la siguiente hipótesis:
H1: el atributo de Emprendimiento de los estudiantes que cursan de último grado de la carrera de empresariales depende, en forma significativa, del comportamiento de la variable Familiares.

Los hallazgos del presente trabajo dejan abierta la posibilidad para estudiar si esta hipótesis se ratifica en el contexto de otros estudiantes de carreras universitarias que cursan los semestres finales de sus respectivas carreras y, finalmente, comprobar si variable Familiares incide directamente sobre la competencia de emprendimiento.

\section{DISCUSIÓN}

El emprendimiento como cualidad humana, estudiada y desarrollada a nivel universitario en su carácter de competencia personal (Castillo y Alejandro, 2016), se 
evidencia desde la formación de pregrado (Farrington, et al., 2012), por una parte, porque el profesor es un actor clave en la sociedad del conocimiento (Pedraja, 2012) $\mathrm{y}$, porque la misma sociedad condiciona la reflexión, el lenguaje y las preguntas que definen, redefinen y transforman progresivamente el rol que le cabe al emprendedor en el escenario de negocios (Bignotti y Le Roux, 2016).

El desarrollo de competencias requiere del docente la capacidad de generar para sus alumnos, un contexto protegido de comprensión y abordaje de la realidad (Morales, et al., 2015). Se precisa que el profesor sea capaz de generar progresivamente en los estudiantes un modelo mental de percepción - comprensión de la realidad y de comportamiento que $(\mathrm{Mu}-$ ñoz-Osuna, 2016), tal como se observa en los resultados de este trabajo, permita a los estudiantes identificar y superar las trazas de temor a la incertidumbre que manifiestan frente a la opción de tomar decisiones de emprendimiento, motivo por el cual, los docentes asumen para si la necesidad de orientar las habilidades de sus estudiantes emprendedores hacia el objetivo que cada quien se trace para sí (Cheril, 2018).

Además, como ha sido referenciado (Robles-Haros, 2016), las universidades forman parte del proceso de enseñanza y desarrollo del talento emprendedor ( $\mathrm{Sa}$ lati, et al., 2018). Si bien porque las universidades se encargan de preparar sus currículums para que sus estudiantes desarrollen cualidades activas de aprendizaje (Sutcliffe, 2005) así como por el propósito que éstas asumen de proveer a sus educandos de las cualidades que les permitan saber cómo afrontar el mundo de los negocios en su rol de emprendedores (Soria, et al., 2016) o como profesionales competentes en su rol de seguidores de quién emprende (Morales y Varela, 2015; Pedraja, 2012).

De acuerdo con los resultados obtenidos en este trabajo, existe una aspiración y meta laboral de emprendimiento de los estudiantes que ratifica los hallazgos realizados por Soria, et al., 2016; Soria-Ba- rreto, et al., 2017 y Salati et al., 2018, que confirman las aspiraciones y metas emprendedoras. Además, se valida la motivación emprendedora que existe en el mercado laboral (Araya y Pedreros, 2013) y que se manifiesta entre los estudiantes (Klein, 2016), como una necesidad de logro (Chinyamurindi, 2016).

Sin embargo es preciso reconocer que el emprendedor busca sus opciones en un terreno inestable o ambiguo (Angulo, 2016; Arquero y Tejero, 2006) que presenta desafíos que deben ser superados mediante el capital que les reporta su aprendizaje y energía de la juventud (Cheril, 2018). Son estas cualidades las que los impulsa a seguir sus caminos para alcanzar progresivamente el rumbo esperado, pero sobre la base de conseguir un adecuado control interno de las situaciones que enfrentan (Bibiano, et al., 2016), por una parte, mediante un aceptable manejo de los grados de tolerancia a la ambigüedad en la toma de decisiones (Arquero y Tejero, 2006) y por otra, porque el sentimiento de logro los impulsa a superar las ambigüedades y les permite a su vez superar el temor al temor al fracaso (Araya y Pedreros, 2013). Claramente, el hecho de superar la aversión natural al riesgo (Angulo, 2016) es un factor determinante para lograr emprender (Bignotti y Le Roux, 2016).

Por otra parte, aunque las universidades se encuentren preparadas académicamente para formar profesionales que movilicen sus talentos de liderazgo emprendedor (Robles-Haros y Esteves-Nenninger, 2016) deben a su vez disponer y potenciar sus propias capacidades de enseñanza que les permitan también formar a los jóvenes profesionales con las habilidades necesarias para ser conductores del futuro (Pedraja, 2012) y puedan crear y dirigir empresas innovadoras.

En términos generales, las variables Edad, Femenino, Masculino, Experiencia y Semestrez que caracterizan a los estudiantes no reportan índices suficientes para determinar una relación estadísticamente significativa. Sin embargo, la variable Familiares presenta un valor de significancia $\left({ }^{*}\right)$ relevante, en consecuen- 
cia, efectivamente ésta es un inductor que activa y promueve las competencias de Emprendimiento de los estudiantes, confirmando los planteamientos de Soria, et al., (2016).

El presente trabajo muestra además que el conocimiento necesario es vital para la labor empresarial generando una ventaja valiosa (Cheril, 2018), sin embargo, parece necesario cuestionarse si la universidad fomenta un verdadero espíritu de emprendimiento en los estudiantes (Robles-Haros y Esteves-Nenninger, 2016). Es por ello que es necesario entender que tal vez el secreto del emprendimiento proviene de su voz latina prendĕre, que significa literalmente tomar o coger una opción que puede ser de riesgo, es decir, tomar una decisión orientada hacia una oportunidad (Araya, 2013) lo cual conlleva la capacidad intrínseca del emprendedor de enfrentar las ambigüedades del entorno (Angulo, 2016) y superar las amenazas.

Por otra parte, puede ocurrir que el emprendedor sea simplemente una persona, hombre o mujer (Ferraz et al., 2014) que toma una decisión arriesgada porque posee algunas cualidades que le reportan energía (Dastourian, et al 2017) y le permiten decidir, arriesgar y tomar decisiones (Chinyamurindi, 2016) o porque tuvieron la opción de ejercitarse en un contexto universitario protegido que les permitió identificar una necesidad social que activó su conducta emprendedora (Araya y Pedreros, 2013) y que asumieron como una oportunidad a pesar de los vaivenes del entorno (Arquero y Tejero, 2006).

\section{CONCLUSIONES}

Del análisis realizado y de la interpretación de los resultados en este trabajo se puede concluir:

1) no se aprecia una mayor predisposición emprendedora entre los estudiantes, por una parte porque no se muestran totalmente convencidos con el emprendimiento y, por otra, porque quienes cuentan con familiares emprendedores, son más adversos al riesgo.
2) Así mismo se aprecia que existe un aceptable nivel de control interno y conciencia de que las decisiones tomadas implican un resultado concordante con las acciones realizadas.

3) En el grupo más joven existe una ligera predisposición a la idea del negocio propio aunque existe temor a la inversión de los ahorros, sin embargo se aprecia que todos los grupos estarían dispuestos a dar todo de si por un negocio propio.

4) Además, sin perjuicio de que las mallas curriculares fomentan el espíritu emprendedor, los estudiantes ecuatorianos, no se encuentran totalmente seguros de emprender.

5) La única dimensión que incide en forma significativa sobre el atributo de emprendimiento es la variable Familiares, en consecuencia, el hecho que el estudiante provenga de una familia de empresarios es, precisamente el inductor de la conducta emprendedora de los estudiantes de último grado.

Se sugiere por último, establecer en lo sucesivo planes de seguimiento de los egresados, así como estudios complementarios que permitan ratificar o rechazar la hipótesis de que efectivamente la variable Familiares es un inductor directo del emprendimiento en el ámbito universitario.

\section{REFERENCIAS}

Angulo, J. M., La Teoría Moderna de Medidas de Riesgo, en el Contexto de la Complejidad, Academia de Ciencias Matemáticas, Físico-Químicas y Naturales de Granada, Universidad de Granada (2016)

Araya, L. y M. Pedreros, Análisis de Las Teorías de Motivación de Contenido: Una Aplicación al Mercado Laboral de Chile del Año 2009, Revista de Ciencias Sociales No 142, 45-61 (2013)

Arquero, J. L. y C. Tejero, Estilos de aprendizaje y tolerancia a la ambigüedad de los estudiantes universitarios. Diagnóstico y Reflexiones ante el EEES, Revista de Enseñanza Universitaria, Número 28, 7-17 (2006)

Bibiano, M., M. Ornelas, J. Aguirre y J. 
Rodríguez, Composición Factorial de la Escala de Locus de Control de Rotter en Universitarios Mexicanos, Formación Universitaria Vol. 9(6), 73-82 (2016)

Bignotti, A. y I. Le Roux. Unravelling the Conumdrum of Entrepreneurial Intention, entrepreneurship education, and entrepreneurial characteristics. Acta Commercii Independent research in the Management Sciences, 16(1), 1-10 (2016)

Castillo Vergara, M. y Á.M. Alejandro. Entrepreneurship Perception in $\mathrm{Hi}^{-}$ gher Education A Comparative Study Among Students, Faculty Menbers And Directors. Revista Latinoamericana de Ciencias Sociales, Niñez y Juventud, 14(1), 221-233 (2016)

Cheril, C., Do Older Or Younger Entrepreneurs Have The Greater Advantage? Forbes Contributors Entrepreneurs Sep. 3, 2012. https://goo.gl/xUnsc7 Acceso: 02 de Abril de (2018)

Chinyamurindi, W. T. A narrative Investigation on The Motivation to Become on entrepreneur among a sample of black entrepreneurship South Africa implications of entrepreneurship carrer development education. Acta Commercii Independent Reserach Journal in The Management Sciences, 1(16), 1-9 (2016)

Contreras Torres, F., J. C. Espinosa Méndez, K. Soria Barreto, A. Portalanza Chavarria, K. Jauregui Machuca y J. A. Omaña Guerrero, Exploring entrepreneurial intentions in Latin American University Students. Internatipnal Journal of Psychological Research, 10(2), 46-59 (2017)

Dastourian, B., H. Kawamorita Kesim N. Seyyed Amiri y S. Moradi, Women Entrepreneurship: Effect of Social Capital, Innovation and Market Knowledge. AD-Mister, 30, 115-130 (2017).

Farrington, S. M., D. J. Venter, C. R. Schrange y V. D. Meer, Entrepreneurial attributes of undergraduate business students: a thrre contry comparison revisited. South African Journal of Economics and Management Sciences, 15(4), 333-351 (2012)

Ferraz Gomez, A., W. Piau Santana, U. Pessoa Araújo y C. M. Fontes Martins. Female Entrepreneurship as Subjet of Research. RBCN Review of Business
Management Subjet Area: Teaching and Research in Management, 319-342 (2014)

Gujarati, D. N. y D.C. Portes, Econometría (5 ed.). México: McGraw-Hill. (2009)

Hernández, R., C. Fernández, y P. Baptista, Metodología de la Investigación. México D. F.: Mc Graw-Hill (2006)

Klein, P. G. Why Entrepreneurs Need Firms, and the Theory of the Firm Needs Entrepreneurship Theory. RA USP Management Journal, 51(3), 323-326 (2016)

Likert, R., A Technique for the Measurement of Attitudes, Archives of Psychology, 140,1-55 (1932)

Lloret, S., A. Ferreres, A. Hernández e I. Tomás, El análisis factorial exploratorio de los ítems: una guía práctica, revisada y actualizada. Anales de psicología, 30(3), 1151-1169 (2014)

Mahadea, D., S. Ramrrop, y T. Zemotir, Assessing entrepreneurship perception of high school learners in Pietermaritzburg, Kwazulu Natal. South african Journal of Economic and $\mathrm{Ma}$ nagement Sciences , 14(1), 66-79 (2011)

Manyaka - Boshielo, S. J. Social Entreprenurship as Way of Developing Suatainable Township Economics. HTS Theological Studies, 73(4), 1-10 (2017)

Morales Castillo, J. D. y M. Varela R. El debate en torno al concepto de competencias. Investigación en educación Medica 4.13 (2015)

Muñoz-Osuna, F. O., A. Medina-Revilla y M. Guillén-Lúgigo. Jerarquización de competencias genéricas basadas en las percepciones de docentes universitarios. Educación Química 2.2 (2016)

Naia, A., R.Baptista, R. Biscaia, C. Januáriop y V.Trigo, Entrepreneurial Intention of Sport Science Students and Theory of Planned Behavior. Motriz: Revista de Educación Fisíca, 23(1), 1421 (2017)

Pedraja, L., Desafíos para el Profesorado en la Sociedad del Conocimiento, ISSN 0718-3305, Ingeniare. Rev. Chil. Ing., 20(1), 136-144 (2012) https://goo.gl/devpgv Acceso: 30 de abril de (2018)

Pérez, C., Técnicas estadísticas multivariantes con SPSS, Ibergarceta Publicaciones, S.L. (2009)

Robles-Haros, B. I. y E. H. Esteves-Nen- 
ninger. Enfoque por competencias: Problemática didácticas que enfrentan el profesorado. Revista Electronica Educare $20.1(2016)$

Rodriguez, O. Entrepreneurship and its analysis in Colombia: A Contextualized Literature Review. Cuadernos de Economía, 34(66), 605-628 (2015)

Salati Marcondes de Moraes, G., E. Sadao Lizuka y M. Pedro. Effects of Entrepreneurial Characteristics and University Environment on Entrepreneurial. RAC , 22(2), 226-248 (2018)

Soria, K., S. Zúñiga y S. Ruiz, Educación e
Intención Emprendedora en Estudiantes Universitarios: Un Caso de Estudio, Formación Universitaria Vol. 9(1), 25-34 (2016)

Soria-Barreto, K., G. Hunores-Marin, P. Gutierrez- Zepeda y J. Gutierrez- Rodriguez, Prior Exposure and Educational Environment Toward Entrepreneurial Intention. Journal of Technology Management \& Innovation, 22(2), 45-58 (2017)

Sutcliffe, N, S.S. Chan y M. Nakayama. Competency Based MSIS Curriculum. Journal of Information System Education 16.3: 301 (2005) 